\title{
Procoor: un sistema para estudiar la geometría espacial
}

\section{Valentina Pellegrini}

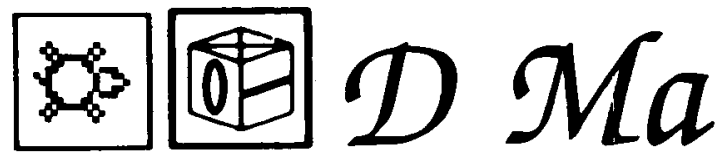

Poder desarrollar programas educativos específicos que pretenden enseñar mejor determinados contenidos del curriculum, yendo más allá de las limitaciones del discurso oral o escrito lineales, es una de las grandes virtudes del ordenador. Se presenta aquí un programa experimental de geometría.

PROCOÓR es un sistema software desarrollado para ofrecer a los maestros y a los alumnos un instrumento apto para explotar al máximo la facultad intuitiva y visual del estudiante al afron. tar el estudio de la geometría espacial. Particularmente se pueden realizar intervenciones didácticas organizadas y sistemáticas para el aprendizaje del contenido geométrico que necesita de una buena percepción espacial como la de la perspectiva de los cuerpos elementales y de la representación de puntos en el sistema de coordenadas cartesianas, cilíndricas y esféricas. Algunos «courseware» (cursos que enseñan cómo elaborarlas) se desarrollaron para la enseñanza de la matemática y gran parte de ellos han dirigido sus pasos al estudio de la geometría. Con estos productos se tiende a valorizar el punto de vista operativo y constructivo de una materia que siempre ha sido tratada en la escuela secundaria superior preferentemente bajo el aspecto axiomático-deductivo. Particularmente el empleo de este software consigue en reencuentro con la geometría a muchos estudiantes dotados de inteligencia «práctica», que tienen necesidad de enfrentarse directamente con todo lo que se les presenta. Se ofrece, sustancialmente, a los estudiantes la oportunidad de practicar las nociones que aprenden a través de la visualización de representaciones complejas comparables entre ellas y obtenidas en breve tiempo, sin desviar la atención del significado real del ejercicio.
Los conceptos geométricos que podemos introducir con el apoyo de un software válido y didáctico disponen de una gran gama de razonamientos: desde los más elementales de construcción conceptual a la definición de los entes geométricos (ver por ejemplo algunos ejercicios que forman parte del paquete MICRO SMILE de Ilea), a aquellos más complejos de la transformación del plano (ver como ejemplo el sistema TRANSFORMAZIONI GEOMETRICHE de Paravia-Ricordi) a la geometría analítica (ver ejemplo del sistema GRAPHING EQUATIONS de Conduit y RETTE de Olivetti), hasta la representación plana de las figuras geométricas del espacio. Es en este último campo en donde se quiere insertar el sistema PROCOOR (prospectiva e sistemi di COORdinate, PREspectiva y sistema de COORdenadas) realizado dentro del proyecto organizado por el Instituto de la Matemática Aplicada del C.N.R. con el tema «Cálculo numérico y progresivo sobre M24» en colaboración con Olivetti.

\section{ESTRUCTURA GENERAL Y OBJETIVOS}

PROCOOR fue concebido como un auxilio didáctico dedicado a la profundización del estudio de la prespectiva de las figuras tridimensionales y de la representación de puntos en el espacio. 
Para alcanzar separadamente los objetivos prefijados, el sistema ha sido dividido en dos módulos principales ( (PREspectiva y dibujo» y «sistema de COORdenadas»), independientes entre ellos, a los cuales se accede a través de un módulo de mando (ver fig. 1).

FIGURA 1

Estructura del sistema

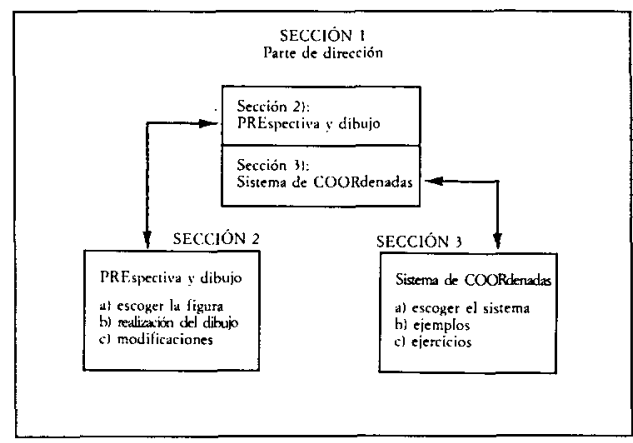

El primer módulo, «PREspectiva y dibujo», relaciona el estudio y la realización en perspectiva de los cuerpos más conocidos a nivel de secundaria superior como el cubo, paralelepípedo, prisma y pirámide.

Una vez definido el cuerpo es posible operar en su representación escogiendo entre varias opciones, presentadas en el menú (ver fig. 2); una de éstas representa una ayuda sobre el uso del sistema.

Figura 2

El menú

\begin{tabular}{|c|}
\hline$* * *$ PRESPECTIVA Y DIBUJO**** \\
\hline$\Lambda * * * * * * * * * * * * *$ Instrucciones $* * * * * * * * * * * * * * * * * * * * * * * * * * * * * * * * *$ \\
\hline B $* * * * * * * * * * *$ Elección de una nueva figura******************** \\
\hline C. $+\star \star \star \star \star \star \star \star \star \star \star \star$ Modificación del punto de vista \\
\hline 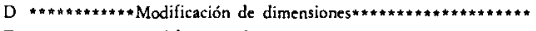 \\
\hline E $* * \star * * \star * * * * * *$ Modificación de posición********************** \\
\hline F \\
\hline 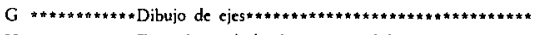 \\
\hline 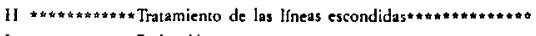 \\
\hline 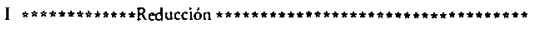 \\
\hline J $\neq * * * * * * * * * * * *$ Ampliación $* * * * * * * * * * * * * * * * * * * * * * * * * * * * * * * *$ \\
\hline K $* * * * * * * * * * * *$ Vuelia a las dimensiones $* * * * * * * * * * * * * * * * * * * * * * * *$ \\
\hline L $\| * * x * * * * * * * *$ Movimiento \\
\hline M $\# \star \star \star \star \star \star \star \star \star \star \star \star \star$ Rotación alrededor del ejc Z Z \\
\hline N \\
\hline
\end{tabular}

Los objetivos que se quieren conseguir con la utilización de este módulo son:

- evidenciar el vínculo entre la representación plana de los cuerpos conocidos y el contenido geométrico que no está en la base, estimulando la intuición del estudiante con una visualización inmediata de la situación espacial abstracta.
- profundizar en el estudio de la propiedad del dibujo de la prespectiva, subrayando las diferentes posibilidades de representación en el espacio de un mismo objeto, según si utilizamos uno o más parámetros.

El segundo módulo de PROOCOR «sistema de COORdenadas», propone el estudio del espacio introduciendo el concepto de representación de puntos (parados o en movimiento) relativo a un sistema de coordenadas. Con este razonamiento nos vienen propuestos ejemplos y ejercicios utilizando el sistema de coordenadas Cartesianas, $\mathrm{Ci}$. líndricas y Esféricas.

El objetivo que se quiere conseguir con este segundo módulo es el de facilitar la comparación entre las diferentes posibilidades de utilización de los tres sistemas arriba citados en relación a la aplicación en el campo científico (navegación, astronomía, etc.).

\section{DESCRIPCION}

Ahora vamos a examinar más detalladamente los dos módulos del sistema para profundizar luego por separado en la finalidad didáctica.

\section{PREspectiva y dibujo}

Con la elección de este módulo se tiene la posibilidad de representar figuras geométricas tridimensionales en un sistema de referencia cartesiana fijado en programa, con origen en el centro del cuerpo (a media altura para la pirámide). Elije la figura y señala dimensiones y punto de vista, es posible escoger el visualizar los otros ejes cartesianos y/o las líneas escondidas del cuerpo. Después de haber obtenido el dibujo de la figura escogida se puede efectuar eventuales modificaciones sobre ella, modificaciones que consiguen: - cambiar el punto de vista y/o una (o más) dimensiones del cuerpo;

- modificar la posición de esta figura en el espacio mediante rotación y traslación (incluso la composición entre ellos);

- modificar la escala (reducción y ampliación) con posibilidad de volver a las dimensiones originales del cuerpo;

- obtener traslaciones en línea recta y rotaciones de 360 grados del cuerpo con ayuda de la animación para el estudio del movimiento.

\section{Sistema de «COORdenadas»}

Este módulo es un sistema de ejercicios que of rece la posibilidad de disponer de ejemplos respecto el modo de empleo del sistema de coordenadas cartesianas, cilíndricas, esféricas. Cada uno de estos sistemas de referencia viene provisto de una descripción teórica respecto a la representa- 
ción de un punto relativo al sistema de coordenadas que examinamos. Elegido el sistema, se puede seleccionar del menú o bien la opción concerniente al ejemplo de representación de puntos o también uno de los ejercicios propuestos de diversa tipología.

Estos están agrupados en tres categorías distintas que permiten:

- representar un punto dada la coordenada;

- valorar la coordenada de un punto dibujado de modo casual en la pantalla. El estudiante debe escoger el grado de dificultad del ejercicio y dispone de tres pruebas al final de las cuales se visualiza el valor real de la coordenada del punto;

- valorar la coordenada de la posición final asumida por un punto en movimiento, conociendo el paso y el número de etapas recorridas. Aunque en este caso se puede escoger el grado de dificultad del ejercicio.

\section{PROPUESTA DE UTILIZACION EN LA ESCUELA}

Se aconseja la utilización de PROCOOR en los dos primeros años de la secundaria superior (lo que para nosotros representaría los primeros cursos de BUP) ya que para los razonamientos introducidos necesitan algunas nociones de geometría del espacio y de la técnica de dibujo de la perspectiva adquirida ya en este nivel escolar. En lo que respecta a la utilización del primer módulo, el maestro debe iniciar el trabajo en clase mostrando a los alumnos algunos ejemplos de perspectiva de los cuerpos sin detenerse en el segundo método en el cual están asignados los datos que han llevado a estos resultados. Por ejemplo, él puede proponer alguna prespectiva del mismo cuerpo según distintos puntos de observación. De este modo el estudiante observará cómo una misma figura se puede «ver» de forma diversa en el espacio.

En esta primera fase del trabajo será tarea del maestro asignar los datos oportunamente para obtener representaciones claras.

En la segunda fase del trabajo el maestro deberá en cambio, dar datos «inadecuados», para provocar la visualización del mensaje de error o de la representación ambigua del cuerpo escogido. De este modo podrá hacer notar que si se escoge un punto de vista demasiado cerca del cuerpo como para dar lugar a una imagen sin significado, el sistema no ejecuta el dibujo, visualizando en su lugar el mensaje correspondiente.

El objetivo de esta segunda fase es claramente el de estimular al estudiante a investigar los motivos que determinan estos resultados.

Después de esta experiencia propedéutica el docente ilustrará cómo hacer ejecutar la correcta construcción de la imagen en prespectiva del cuerpo a calcular, ayudando al estudiante al escoger los datos y explicando por grados, la solución del problema propuesto. Este tipo de ejercicios asistido por el maestro durarán hasta que el alumno haya adquirido suficiente destreza en los razonamientos; únicamente al llegar a este punto se sentirá protagonista de la lección y por consiguiente más motivado y atento al enfrentarse con la materia de estudio.

Después de la primera fase de la experiencia se podrá profundizar teóricamente en el concepto de prespectiva y estudiar el método geométrico de base usado para realizarla.

Es aconsejable utilizar las otras opciones ofrecidas en el sistema sólo sucesivamente, cuando modifica la posición del cuerpo, al cambiar la escala, y la modificación de los datos iniciales. Esta última fase del trabajo, precedida de las lecciones explicativas del maestro, conseguirá subrayar posteriormente la propiedad geométrica del dibujo de la prespectiva y las diferentes posibilidades de representación de un mismo objeto en el espacio con la única modificación de algún parámetro (ver ejemplo 1).

Todo lo propuesto para el primer módulo sirve para el segundo, se aconseja el recorrido didáctica que sigue las fases: «ejemplo», «teoría» y «uso del sistema».

En este sentido es conveniente que el maestro inicialmente se sirva de los ejemplos propuestos por el sistema sobre la representación de puntos entre los tres distintos sistemas de coordenadas. Una vez aclarados los conceptos base, el alumno podrá examinar el mismo razonamiento por via teórica. Particularmente el maestro deberá introducir el sistema de coordenadas cartesianas, cilíndricas y esféricas, explicando los motivos de la elección por los que se trabaja con un sistema más difícil que otro en los diversos campos de aplicación.

Superada esta fase se les propone a los alum. nos los ejercicios presentados en el menú.

Es conveniente, al menos inicialmente, que el estudiante elija la opción en la que tenga la posibilidad de asignar la coordenada de un punto; siguiendo luego el programa podrá verificar si sus cálculos son correctos o no.

Luego examinaremos otros ejercicios más complejos que nos pedirán un mediano conocimiento del sistema de coordinadas en el espacio y una buena percepción de la perspectiva, por ejemplo si consideramos los ejercicios relativos a la valoración de las coordenadas de un punto fijo, dibujado casualmente en la pantalla.

Finalmente el maestro podrá proponer análogos ejercicios más complejos, en donde vengan representados puntos en movimiento para los que se tendrá que asignar la coordenada del punto de llegada. (Ver ejemplo 2).

\section{Ejemplo 1}

\section{Problema}

Dibujar un prisma pentagonal, con radio de base 3 unidades y altura 7 unidades, visto en pres- 
pectiva del punto de vista $(7,8,9)$, en un sistema de ejes cartesianos.

Reducir el cuerpo con factor de reducción de 3 unidades, luego trasladarlo de $(0,05)$ y girarlo 90 grados sobre el plano yz y dibujar el cuerpo que se obtiene.

Actividad prevista:

Escoger la opción relativa a la realización de perspectiva; responder secuencialmente a las cuestiones puestas, en base a los términos del problema:

ELECCION DE LA FIGURA: responder 3 (relativa al prisma)

NUM. LADOS BASE: 5

RADIO DE BASE: $3 \mathrm{u}$ (radio circunferencia inscrita)

ALTURA: $7 \mathrm{u}$

PUNTO DE VISTA: $(7,8,9)$

Interacción con el alumno:

Comparar el menú principal (ver fig. 2). Se escoge la opción $\mathrm{G}$ (dibujo ejes), en lugar de la opción $\mathrm{F}$ (relativa al dibujo). Se obtendrá en la pantalla el dibujo del prisma dado: (fig. 3).

Volvemos al menú principal (RETURN) y se escoge la opción referente a la reducción. Se le asigna el valor 3 al factor solicitado de la reducción. De esta manera se realizará la reducción de la figura dada (fig. 4).

Volvemos al menú principal (RETURN) y se elige la opción de Modificación de la posición.

Para la traslación: del menú que se presenta se selecciona la opción correspondiente y se introduce los datos $(0,05)$.

Para la rotación: responder SÍ a la petición para la modificación, luego se escoge 3 y se introduce 90 como ángulo de rotación.

Se responde NO para otras modificaciones y para ver contemporáneamente la figura original y modificada. En este punto se selecciona la opción F para obtener el dibujo previsto (fig. 5).

\section{FIGURA 3}

Representación del prisma

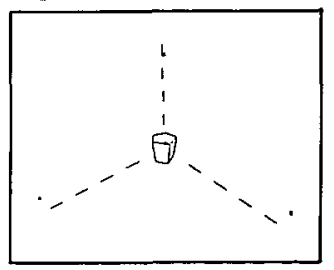

FIgURA 4

Reducción del prisma

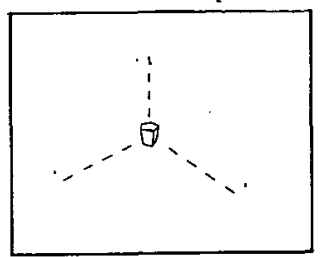

\section{Figura 5}

Traslación y rotación del prisma

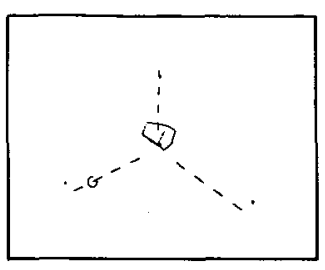

\section{Ejemplo 2}

\section{Problema:}

Asignar los valores que el punto, que se mueve en línea recta, interceptará en uno de los ejes de las coordenadas.

Actividad prevista:

Seleccionar la opción concerniente a la coordenada cartesiana.

Interacción con el alumno:

Comparar el menú principal que propone una serie de ejercicios.

Escoger una de las opciones que vienen visualizadas en una serie de posibilidades de elección según el grado de dificultad con que se quiere seguir el ejercicio.

En el ejerccio 5, una vez escogido el ejemplo 0 se representará el punto en movimiento (fig. 6).

Cuando terminemos esto, asignaremos los valores exactos que están retenidos exactos por las coordenadas de las posición final del punto. En tal caso se obtendrá en la pantalla un mensaje de aprobación como se ve en la fig. 7.

En caso de error se visualizará la coordenada exacta.

\section{Figura 6}

Representación de un punto en movimiento:

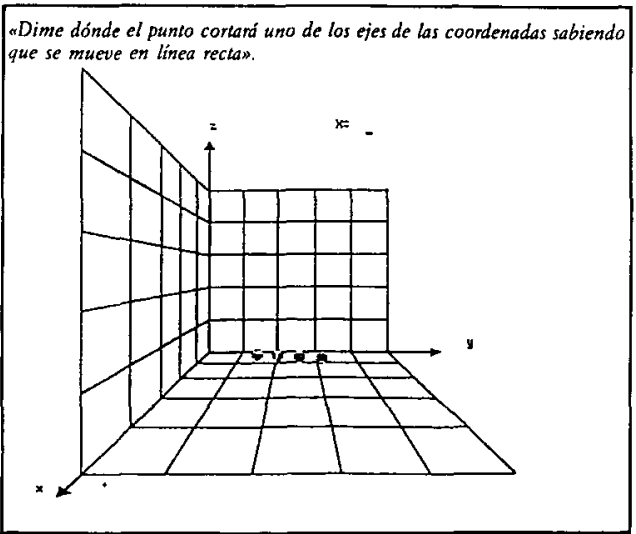


Figura 7

Ejemplo de asignación de valores a las coordenadas

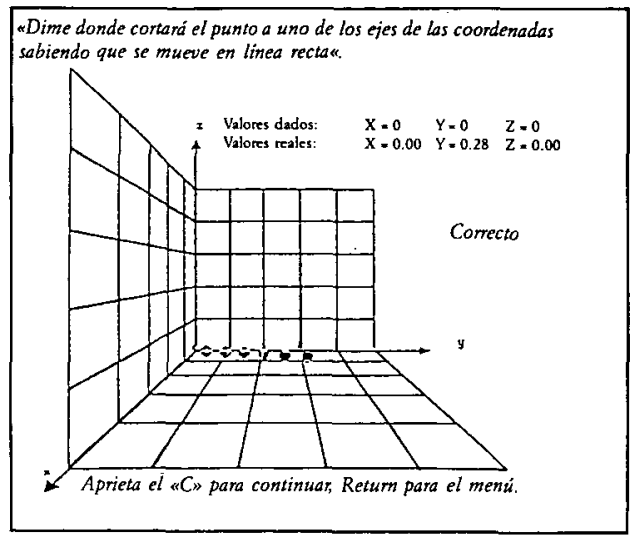

\section{FICHA SOFTWARE}

Programa: PROCOOR

Autora: Valentina Pellegrini

Año: 1987

Ordenador: Olivetti M24

Configuración: $\quad-256 \mathrm{~Kb}$

- MS-DOS

- Ver.2.11

- GW-Basic

Composición: un disquette, instrucciones

Materia: Matemática

Tema: Prespectiva y sistema de coordenadas

Objetivos: Comprender la relación entre la representación plana de situaciones tridimensionales en el contenido geométrico de base.

Nivel escolar: Primer bienio de la secundaria superior (los dos primeros años de BUP).

Proocoor: un sistema para estudiar

la geometría espacial

Valentina Pellegrini

CL\&E, 1992, 13, pp. 89-93

Datos de la autora: Investigadora del Instituto para las Matemáticas Aplicadas.

Dirección: Instituto para las Matemáticas Aplicadas del CNR de Génova.

Artículo original: Procoor: un sistema per lo studio della geometria dello spazio. Golem, N. 3 marzo 1990, pp.6-11. Reproducido con autorización. Traducción de Lola Abelló.

(C) De todos los artículos deberá solicitarse por escrito autorización de CL\&E y de los autores para el uso en forma de facsímil, fotocopia o cualquier otro medio de reproducción impresa. CL\&E se reserva el derecho de interponer las acciones legales necesarias en aquellos casos en que se contravenga la ley de derechos de autor. 